\title{
Bieg, Peter: Mediensportarten abseits des Fußballs. Basketball in Deutschland: eine explorative Fallstudie zum Kampf um Platz zwei im Sport. Sportkommunikation, Band 16
}

\author{
Köln: Herbert von Halem 2019. 264 Seiten. Preis: $€$ 25,99 \\ (e-book)
}

\author{
Mark Ludwig \\ Online publiziert: 24. September 2020 \\ (C) Der/die Autor(en) 2020
}

Wenn in Deutschland über Sport berichtet und geforscht wird, dann geht es oft um „König Fußball““. Studien, die sich vertiefend mit einzelnen anderen Sportarten auseinandersetzen, gibt es bisher eher wenige. Peter Bieg ist diesen Weg gegangen und nimmt in seiner in der Reihe „Sportkommunikation“ erschienenen Dissertation die Sportart Basketball in den Blick. Maßgeblich geht es bei ihm um die Frage, wie sich Basketball medial erfolgreich im „Kampf um Platz zwei“ im SportartenRanking etablieren könnte. Bieg, der als Sportjournalist im Feld der BasketballBerichterstattung tätig war, greift die in Forschung wie Praxis vieldiskutierte Frage auf, wie Sportarten mediale Aufmerksamkeit generieren und optimieren können - mit dem Blick auf die bisher in der deutschsprachigen Forschung eher wenig beachtete Sportart Basketball setzt er dabei einen neuen Impuls.

Der Band setzt jedoch nicht nur thematisch neue Akzente. Von besonderem Forschungsinteresse ist die Arbeit auch, da sie mit dem Rückgriff auf Bourdieus Sozialtheorie einen neuen Versuch unternimmt, die „Untersuchung der erfolgskritischen Attribute" von Sportarten theoretisch zu fundieren und auf dieser Grundlage empirisch zu erfassen, ,wie sich ihr Kapital zusammensetzt und die Position im Feld des Sports bestimmt.“ Bieg versteht in Rückgriff auf Bourdieu Sportarten als ,kollektive Akteure mit eigenem Habitus und einer spezifischen Zusammensetzung von Kapital“. Der Transfer von Begrifflichkeiten und Überlegungen Bourdieus, der sich in einzelnen Beiträgen auch selbst bereits dem Sport gewidmet hatte, auf das Feld der Sportkommunikation liefert hierbei weiterführende theoretische Anregungen, die sich auf Grund der klar strukturierten Darstellung auch für Analysen anderer Sportarten sinnvoll nutzen ließen.

\footnotetext{
Dr. M. Ludwig $(\bowtie)$

Institut für Kommunikations- und Medienforschung, Deutsche Sporthochschule Köln, Am Sportpark Müngersdorf 6, 50933 Köln, Deutschland

E-Mail: ludwig@dshs-koeln.de
} 
Etwas schade ist, dass die Diskussion naheliegender Verknüpfungen zu bereits bestehenden Ansätzen etwas zu kurz kommt - so beispielsweise zu den konsumkapitaltheoretisch geprägten Arbeiten von Schellhaaß, die unter anderer Perspektive, aber mit teils ähnlichen Begrifflichkeiten zu vergleichbaren inhaltlichen Überlegungen kommen. Das geschieht im Band „Wie kommt der Sport ins Fernsehen?“ von Schellhaaß und Hafkemeyer auch mit Blick auf den Basketball. Zudem wäre eine ausführlichere Einbeziehung internationaler Perspektiven sicherlich zielführend, insbesondere mit vergleichendem Blick auf den sehr erfolgreich etablierten USamerikanischen Basketball.

Methodisch setzt Bieg in seiner Arbeit im Sinne einer ersten Exploration des Feldes auf einen qualitativen Ansatz. In einer Expertenbefragung werden insgesamt 19 Experten (u. a. Sportredakteure, Funktionäre, Sponsoren) befragt, ergänzend wurden qualitative Dokumentenanalysen (u. a. journalistische Beiträge, Bücher, Statistiken) durchgeführt. Bieg gelangt auf dieser Basis zu detaillierten Einblicken, hierzu tragen auch die durchgängig hochwertigen Gesprächspartner bei. Zudem kommt hier auch Biegs eigene journalistische Erfahrung zum Tragen.

In der darauf aufbauenden Analyse kann Bieg zeigen, dass Basketball zwar eine Sportart mit hohem ,,inkorporierten Kulturkapital“ darstellt, also grundsätzlich telegen ist, diesen Trumpf aber nicht ausspielen kann, da es laut Bieg an Stars und kontinuierlichem Erfolg im deutschen Basketball fehle. „Erst wenn dauerhaft Erfolge und Stars als sich wechselseitig bedingende Merkmale sowie langfristig eine stärkere gesellschaftliche Verankerung und Tradition von Basketball im Raum des Sozialen sichergestellt werden, kann das inkorporierte Kulturkapital der Sportart im Kampf um Handlungsspielraum zusätzlich trumpfen“, folgert er. Die in jüngerer Zeit in der Sportkommunikations-Forschung diskutierten Möglichkeiten der Medialisierung von Sportarten sieht Bieg hingegen eher als ,Zünglein an der Waage“.

Insgesamt stellt Bieg eine Studie vor, die deutlich macht, dass auch der Blick auf Sportarten jenseits des dominierenden Fußball-Sports lohnen kann - unter anderem weil in entsprechenden Detailanalysen spezifische Herausforderungen einzelner Sportarten differenziert in den Blick geraten. Basketball-Interessierte erhalten so einen detaillierten Einblick in eine bisher in der deutschsprachigen sportkommunikationswissenschaftlichen Forschung nur randständig beachtete Sportart. Einen Mehrwert bietet Biegs Arbeit zudem, weil sie ein neues theoriebasiertes Analyseraster zur Verfügung stellt, das einen stärker geistes- und sozialwissenschaftlich geprägten Blick auf die Thematik erlaubt.

Vor dem Hintergrund der hohen Praxisrelevanz der zentral diskutierten Frage, welche Merkmale von Mediensportarten letztlich die erfolgsentscheidenden sind, ist die gut lesbare Studie dabei nicht nur von wissenschaftlichem Interesse, sondern sicher auch für Medienpraktiker eine anregende Lektüre.

Funding Open Access funding enabled and organized by Projekt DEAL.

Open Access Dieser Artikel wird unter der Creative Commons Namensnennung 4.0 International Lizenz veröffentlicht, welche die Nutzung, Vervielfältigung, Bearbeitung, Verbreitung und Wiedergabe in jeglichem Medium und Format erlaubt, sofern Sie den/die ursprünglichen Autor(en) und die Quelle ordnungsgemäß nennen, einen Link zur Creative Commons Lizenz beifügen und angeben, ob Änderungen vorgenommen wurden. 
Die in diesem Artikel enthaltenen Bilder und sonstiges Drittmaterial unterliegen ebenfalls der genannten Creative Commons Lizenz, sofern sich aus der Abbildungslegende nichts anderes ergibt. Sofern das betreffende Material nicht unter der genannten Creative Commons Lizenz steht und die betreffende Handlung nicht nach gesetzlichen Vorschriften erlaubt ist, ist für die oben aufgeführten Weiterverwendungen des Materials die Einwilligung des jeweiligen Rechteinhabers einzuholen.

Weitere Details zur Lizenz entnehmen Sie bitte der Lizenzinformation auf http://creativecommons.org/ licenses/by/4.0/deed.de.

Dr. Mark Ludwig ist wissenschaftlicher Mitarbeiter am Institut für Kommunikations- und Medienforschung an der Deutschen Sporthochschule Köln. 